\title{
从科技论文著者群体的年龄分布看我国科技队伍的 构成状况
}

\section{金碧辉 李玲朱献有 刘䈗敏阳宁晖苑玉兰陆耘汪志南}

(中国科学院文献情报中心, 北京 100080. E-mail: csci@mail.las.ac.cn)

\begin{abstract}
摘要 以中国科学引文数据库(CSCD)1995 1999 年的数据为样本, 统计分析了我国科研队伍年龄分布 的历史状况及演变趋势, 观察到我国特殊历史时期所形成的 “人才双峰结构” 在一种非常有节律的 “长 消” 运动中出现了转机, 并对新时期的人才流失现象进行了思考. 运用科学创造最佳年龄的理论, 预测 在未来 10 年间, 我国科技论文高产年龄区域将与科学创造的最佳年龄趋于一致, 这将为我国科学事业 进入一个快速发展时期奠定坚实的人才基础. 指出我国科技队伍的稳定与建设必须以制度来保证, 按 照市场经济规律的要求加快建立新的用人机制和激励机制是时代提出的迫切要求.
\end{abstract}

\section{关键词 年龄结构 科技人才 人才政策 人才断层 论文产出力}

科技人才是科学发展过程中最活跃的主体因素. 一个国家科学研究能力的大小以及科学成果向社会 经济领域转移和辐射能力的大小是与一个国家科技 人才队伍的知识结构、能力结构以及年龄结构密切相 关的. 由于历史的原因, 我国科技队伍的年龄结构一 直是科研管理部门所关注的问题. 早在 1985 年中国 科学院机关计算机室曾对 1270 项研究成果完成人的 年龄做过统计. 1993 年胡小元等人 ${ }^{[1]}$ 通过问卷调查, 对 2426 名论文著者的年龄进行了统计. 这两项统计 虽然方法不同, 对象迥异, 但是却得到了非常相似的 结果：明显存在人才断层现象. 1985 年统计的断层在 36 40 岁, 而 8 年后胡小元等人的统计, 断层明显增 大, 处在 32 47 岁; 论文产出的高峰区处在 49 58 岁 年龄段, 较 1985 年的统计后移 8 年左右.

本文利用 1995 1999 年中国科学引文数据库大 样本量的数据, 试图从著者的年龄结构出发, 运用文 献计量学的方法, 分析我国科研队伍年龄分布的历 史状况及演变趋势, 旨在帮助管理部门摸清目前我 国科研队伍的年龄结构和存在的问题, 从而制定出 合理的人才政策, 优化人才结构, 建设一支能够适应 21 世纪科技竞争的人才队伍.

\section{1 统计源与基本数据}

本项研究的数据取自中国科学引文数据库 (Chinese Science Citation Database, 简称 CSCD). CSCD 是在国家自然科学基金和中国科学院共同资助下, 由 中国科学院文献情报中心研建的一个大型数据库. 该 数据库收录了中国出版的中英文科技核心期制, 1989
1995 年为 315 种，1996 1998 年为 582 种，1999 年为 633 种, 其学科范围覆盖了数学、物理学(力学)、化 学、天文学、地球科学、生物学、农林科学、医药卫 生、工程技术、环境科学和管理科学等领域, 是我国 科技评价和文献检索的重要工具. 本研究选用了 CSCD 1995 1999 年 5 年的数据, 得到其中附有年龄信 息的著者共 103760 人次. 统计样本中的最小年龄为 18 岁, 最大年龄为 90 岁, 比较全面地反映了 1995 1999 年 5 年间活跃在我国各学科领域的科学家的年龄状况.

对于数据, 有两点需要作特别说明. 第一, 本项 研究的数据属于随机统计样本. 据统计, 在 CSCD 1999 年的 633 种来源期刊中, 有 487 种期刊附有作者 年龄信息, 因此, 本文的期刊统计源, 严格地说, 是 CSCD 来源期刊中附有作者年龄信息的那些期刊. 第 二, 由于绝大多数期刊只提供第一作者的信息, 因此, 本项研究主要是基于第一作者的年龄信息, 部分研 究包括了合作者的信息.

对 103760 个著者人次的年龄样本，分别计算各年 度每一年龄著者群产出论文数占总数的百分比, 得到 1995 1999 年我国科技论文著者年龄分布状况的基本 数据(表 1). 我们依照基本数据, 以著者年龄为横轴, 以每一年龄产出论文所占百分比为纵轴, 绘制出 1995 1999 年各年度我国科技论文著者年龄分布图(图 1). 需要说明的是, 由于样本年龄跨度太大(18 90 岁), 而其中 22 岁之前的人员论文产出力极低, 65 岁后曲线 逐渐贴近横轴, 且没有起伏变化, 受长度的限制, 图 中仅截取有22 65 岁之间的曲线进行分析. 
图 1 反映了我国科技论文产出能力中最活跃的 布中的典型性和代表性，并与全部著者的年龄分布 群体(第一著者)在 1995 1999 年的年龄分布状况. 为 进行比较，我们以 1995 和 1999 年提供全部著者年龄 说明第一著者年龄分布在我国科技论文著者年龄分＼cjkstart信息的期刊为统计样本，得到图 2.

表 1 1995 1999 年我国科技论文著者(第一著者)年龄分布状况/\%

\begin{tabular}{|c|c|c|c|c|c|c|c|c|c|c|c|}
\hline 年龄 & 1995 年 & 1996 年 & 1997 年 & 1998 年 & 1999 年 & 年龄 & 1995 年 & 1996 年 & 1997 年 & 1998 年 & 1999 年 \\
\hline 18 & 0.01 & 0.00 & 0.00 & 0.00 & 0.00 & 55 & 2.72 & 2.16 & 1.52 & 1.29 & 1.04 \\
\hline 19 & 0.00 & 0.00 & 0.00 & 0.00 & 0.00 & 56 & 3.21 & 2.14 & 1.81 & 1.33 & 0.98 \\
\hline 20 & 0.00 & 0.05 & 0.02 & 0.01 & 0.02 & 57 & 3.62 & 2.75 & 1.92 & 1.51 & 1.20 \\
\hline 21 & 0.00 & 0.03 & 0.06 & 0.07 & 0.03 & 58 & 3.25 & 3.03 & 2.34 & 1.80 & 1.60 \\
\hline 22 & 0.25 & 0.16 & 0.12 & 0.16 & 0.19 & 59 & 3.02 & 2.68 & 2.45 & 1.62 & 1.61 \\
\hline 23 & 0.47 & 0.56 & 0.60 & 0.49 & 0.52 & 60 & 2.39 & 2.14 & 1.89 & 1.28 & 1.35 \\
\hline 24 & 1.07 & 1.26 & 1.50 & 1.25 & 1.33 & 61 & 1.39 & 1.35 & 1.15 & 0.91 & 1.40 \\
\hline 25 & 1.93 & 1.92 & 2.26 & 2.59 & 2.59 & 62 & 1.38 & 1.23 & 1.07 & 0.67 & 1.19 \\
\hline 26 & 3.00 & 2.78 & 3.13 & 3.57 & 3.82 & 63 & 0.76 & 0.82 & 0.90 & 0.63 & 0.95 \\
\hline 27 & 3.60 & 3.66 & 3.57 & 3.93 & 4.47 & 64 & 0.45 & 0.68 & 0.61 & 0.47 & 0.73 \\
\hline 28 & 3.89 & 3.98 & 4.18 & 4.81 & 4.39 & 65 & 0.73 & 0.40 & 0.52 & 0.46 & 0.69 \\
\hline 29 & 4.60 & 3.41 & 4.13 & 4.27 & 4.21 & 66 & 0.47 & 0.46 & 0.32 & 0.31 & 0.43 \\
\hline 30 & 6.27 & 5.31 & 4.11 & 4.70 & 4.15 & 67 & 0.37 & 0.31 & 0.42 & 0.26 & 0.39 \\
\hline 31 & 6.00 & 6.22 & 4.75 & 3.78 & 4.30 & 68 & 0.28 & 0.31 & 0.30 & 0.20 & 0.23 \\
\hline 32 & 6.95 & 6.83 & 6.43 & 5.68 & 3.56 & 69 & 0.09 & 0.21 & 0.20 & 0.19 & 0.30 \\
\hline 33 & 4.71 & 6.96 & 7.25 & 6.55 & 5.04 & 70 & 0.22 & 0.12 & 0.22 & 0.09 & 0.19 \\
\hline 34 & 2.18 & 5.15 & 7.24 & 7.36 & 5.85 & 71 & 0.16 & 0.16 & 0.12 & 0.08 & 0.14 \\
\hline 35 & 2.18 & 2.47 & 4.87 & 7.35 & 6.96 & 72 & 0.10 & 0.12 & 0.07 & 0.06 & 0.15 \\
\hline 36 & 1.87 & 2.22 & 2.21 & 4.65 & 6.69 & 73 & 0.10 & 0.05 & 0.07 & 0.04 & 0.08 \\
\hline 37 & 2.12 & 1.77 & 1.91 & 2.20 & 4.19 & 74 & 0.12 & 0.10 & 0.07 & 0.04 & 0.09 \\
\hline 38 & 2.53 & 2.07 & 1.75 & 2.03 & 2.19 & 75 & 0.10 & 0.01 & 0.04 & 0.03 & 0.06 \\
\hline 39 & 1.86 & 2.38 & 2.05 & 1.76 & 1.92 & 76 & 0.12 & 0.07 & 0.05 & 0.04 & 0.05 \\
\hline 40 & 1.70 & 2.22 & 2.55 & 2.23 & 1.55 & 77 & 0.06 & 0.05 & 0.05 & 0.02 & 0.04 \\
\hline 41 & 1.32 & 1.44 & 2.10 & 2.29 & 1.90 & 78 & 0.04 & 0.05 & 0.04 & 0.03 & 0.04 \\
\hline 42 & 0.82 & 1.40 & 1.69 & 2.03 & 2.36 & 79 & 0.04 & 0.03 & 0.02 & 0.02 & 0.03 \\
\hline 43 & 0.95 & 0.87 & 1.40 & 1.63 & 1.93 & 80 & 0.01 & 0.03 & 0.02 & 0.02 & 0.01 \\
\hline 44 & 0.59 & 0.82 & 0.98 & 1.18 & 1.35 & 81 & 0.00 & 0.04 & 0.01 & 0.02 & 0.01 \\
\hline 45 & 0.67 & 0.80 & 0.74 & 1.01 & 1.24 & 82 & 0.03 & 0.01 & 0.00 & 0.01 & 0.02 \\
\hline 46 & 0.63 & 0.79 & 0.66 & 0.75 & 0.97 & 83 & 0.01 & 0.01 & 0.01 & 0.01 & 0.02 \\
\hline 47 & 0.81 & 0.86 & 0.77 & 0.81 & 0.85 & 84 & 0.01 & 0.01 & 0.01 & 0.00 & 0.01 \\
\hline 48 & 1.20 & 1.03 & 0.86 & 0.77 & 0.61 & 85 & 0.00 & 0.01 & 0.01 & 0.01 & 0.02 \\
\hline 49 & 1.76 & 1.19 & 0.66 & 0.68 & 0.70 & 86 & 0.00 & 0.01 & 0.00 & 0.01 & 0.00 \\
\hline 50 & 2.08 & 1.85 & 1.22 & 0.88 & 0.68 & 87 & 0.00 & 0.01 & 0.00 & 0.00 & 0.01 \\
\hline 51 & 1.52 & 1.59 & 1.56 & 0.89 & 0.67 & 88 & 0.00 & 0.00 & 0.00 & 0.00 & 0.00 \\
\hline 52 & 1.20 & 1.26 & 1.75 & 1.62 & 0.88 & 89 & 0.00 & 0.00 & 0.00 & 0.01 & 0.00 \\
\hline 53 & 1.79 & 1.27 & 1.36 & 1.35 & 1.45 & 90 & 0.00 & 0.00 & 0.00 & 0.00 & 0.00 \\
\hline 54 & 2.20 & 1.86 & 1.27 & 1.20 & 1.37 & & & & & & \\
\hline
\end{tabular}

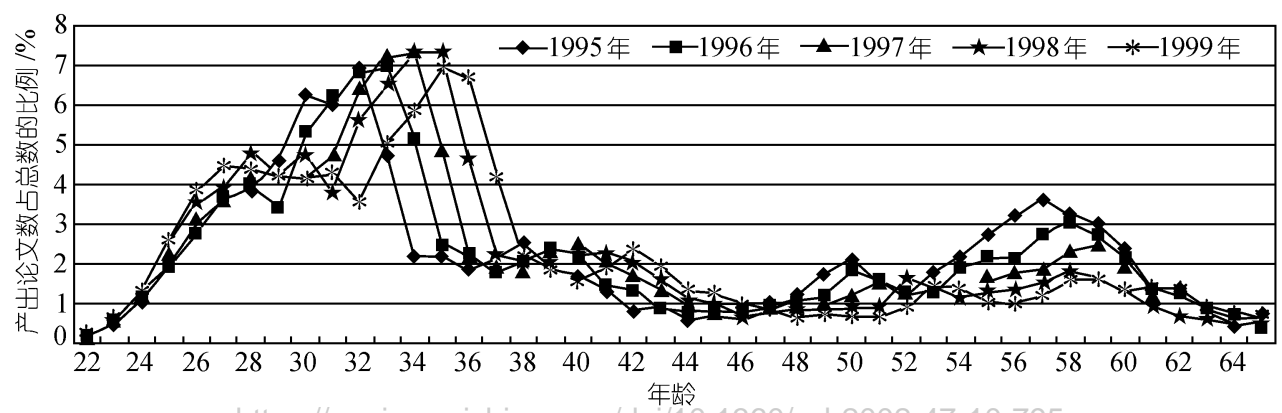

图1 1995 1999 年我国科技论文著者(第一著者)的年龄分布 


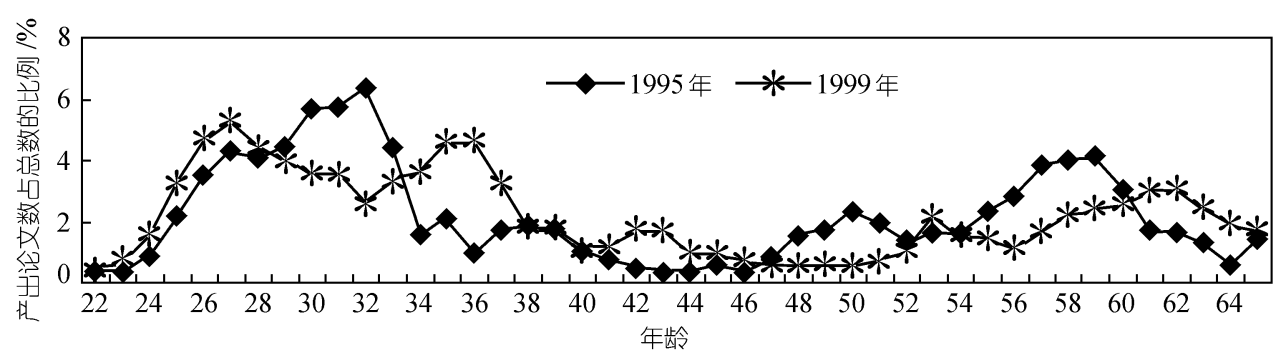

图 21995 和 1999 年我国科技论文著者(全部著者)的年龄分布

将图 1 与图 2 比较, 1995 和 1999 年两条曲线的 形态非常相似: 首先两图均有大小 5 个波峰, 且波峰 的年龄位置基本一致. 以 1999 年的数据为例，第 1 波峰在 26 27 岁，第 2 波峰在 35 36 岁, 第 3 波峰在 42 43 岁, 第 4 波峰在 53 岁, 第 5 波峰在 59 62 岁. 其 次, 从两图的发展趋势来看, 均表现为青年峰在生长, 而老年峰在衰减. 因此我们认为, 第一著者的年龄分 布在我国科技论文产出能力的年龄结构中具有典型 性和代表性.

\section{2 我国科技论文著者群体的年龄分布特征}

由图 1 所表现的曲线形态和数据的分析来看, 我 国科技论文著者群体的年龄分布具有如下几个特征:

（i ）双峰并立现象逐渐演变成主次峰结构

1995 年的著者年龄曲线呈现出一种双峰结构: 第 1 个高峰分布在 29 33 岁, 是改革开放后培养出的 青年人; 第 2 个高峰分布在 55 59 岁, 是 “文革” 前 培养出的老一代科研人员, 两峰之间的低谷比较明 显, 分布在 42 47 岁, 恰好是由 “文革” 造成的一个 人才断层. 此后几年, 著者的年龄结构出现了很有节 律的运动变化. 第 1 个峰值在保持着强劲势头的同时 逐年后移, 而与之表现为联动关系的第 2 个峰值却在 58 60 岁的年龄区域中逐年衰减.

从双峰移动的节律性变化中, 我们可以向前推测, 目前处于第 2 峰的著者群体(老年群体)在 “文革” 结束 以后的改革开放初期是我国科技论文的主要产出群体 (当时的中青年群体), 随着改革开放后新一代青年人才 的脱颖而出, 大约在 20 世纪 90 年代初, 曾经出现两峰 并举, 平分秋色的局面. 而后, 青年群体迅速崛起, 成 为主峰. 由此可以断定, 我国特殊历史时期所形成的 “双峰并立现象” 在一种非常有节律的 “长消” (生长 和消失)运动中逐步演变成为主峰和次峰的结构.

(ii) 第一峰值年龄向最佳科学创造峰值年龄逼近 科学创造最佳年龄的理论认为, 人才成长具有 一定的规律性. 人一生的各个阶段中科学研究能力
是变化的, 一般来说经历了由低到高, 达到顶峰, 随 后逐渐衰弱的过程, 表现为各年龄段科研成果产出 力的不均衡性. 1979 年我国科学学专家赵红州 ${ }^{[2]}$ 提出 了科学创造最佳年龄在 25 45 岁之间, 峰值为 37 岁; 国外的研究成果也同样表明, 科研人员在 36 45 岁的 年龄段上, 创造能力处于较高的水平; 最近的研究表 明, 在大科学时代, 由于科学研究复杂程度提高, 最 佳创造年龄产生了后移.

图 1 表明, 我国科技论文产出力的第 1 个峰值具 有比值稳定和逐年向后平移的特征. 5 年之中第 1 峰 值一直保持在 $7 \%$ 左右, 相当稳定. 统计数据表明, 各年中处于产出力高峰的是同一个群体, 每过 1 年, 该群体的峰值年龄随之递增 1 岁. 这说明我国科技论 文高产年龄群体正在走向成熟, 在向最佳科学创造 峰值年龄逼近.

（iii）在青年群体中有形成新的低谷的趋势

从图 1 中我们可以观察到 1995 年出现了一个新 的低谷, 虽然与第 1 峰相比, 落差不是很大, 但是, 它在此后的几年中持续地以低谷状态匀速后移值得 我们思考. 1995 和 1999 年两年的数据相比, 29 岁的产 出力下降比率为 $10.22 \%, 30$ 岁的产出力下降比率为 $21.69 \%, 31$ 岁的产出力下降比率为 $16.50 \%, 32$ 岁的产 出力下降比率为 $15.25 \% .29$ 32 岁年龄段科研人员论 文产出力逐年下降, 有形成新的低谷的趋势.

(iv) 引人注目的 3 大群体的发展态势

图 1 是根据著者发表论文时的年龄而统计的曲线 分布, 它所展示的是某一年论文著者的年龄分布. 如 果我们将数据固定在著者的出生年上, 考察一下某一 年出生的著者的论文产出数量的分布, 便得到图 3 .

从图 3 可以看到, 各个出生年的著者群体的论文 产出力具有不同的增长速度. 从曲线的变化情况来 看, 明显存在 3 个具有代表性和典型性的群体, 即上 升群、稳定群和衰减群.

年升群: 1968 年后出生的著者群体, 其论文产出 


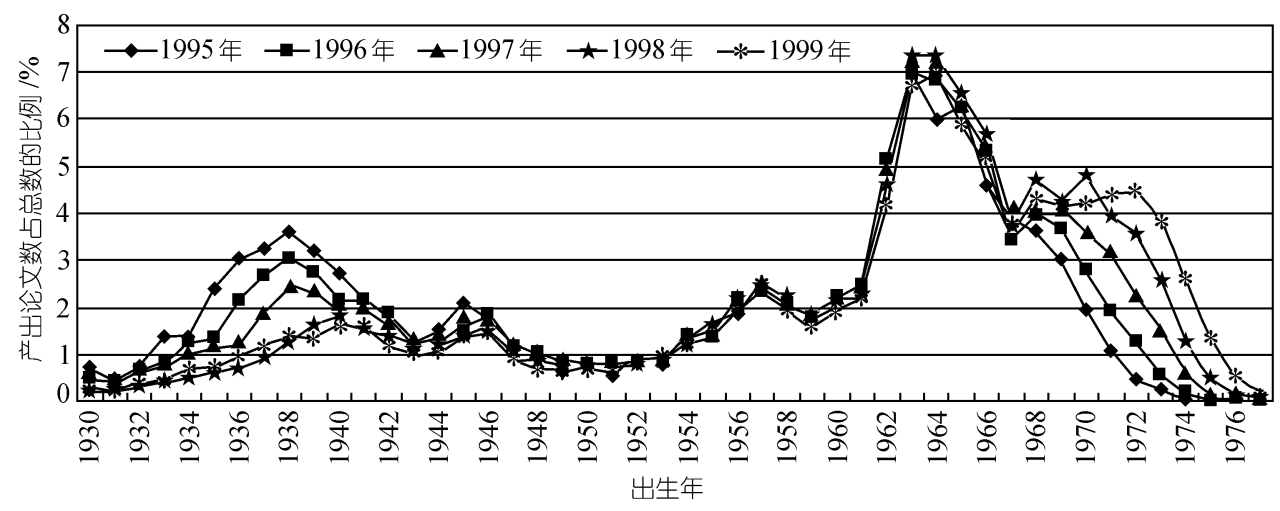

图 31995 1999 年我国科技论文著者的出生年分布

力逐年增长, 是最活跃和最富有成长性的上升群体, 其中以 1971 年为拐点, 1971 年后出生的著者群体的 论文产出力年年持续快速增长. 然而, 在这一群体中, 1968 1970 年出生的著者群的论文产出力虽然也在增 长, 但速度减缓, 1999 年甚至出现了负增长.

稳定群: 1943 1967 年出生的著者群体, 5 年之中论 文产出力保持了几乎是惊人的稳定状态, 其中构筑最 高峰的著者群体, 即在 1963 1966 年出生的著者又形 成了稳定高产群体. 3 个群体中, 稳定著者群的年代跨 度最大, 是目前我国科研队伍的中坚和骨干方阵.

衰减群: 1933 1942 年出生的著者群体, 由于处 在退休年龄段, 论文产出力逐年衰退. 这一现象不仅 符合人的生理现象，也与人才发展规律相吻合. 之所 以这一年龄段的著者还能在曲线图中形成一个动态 变化的峰势, 是因为老一代科学精英以毕生贡献为 人生境界, 还在为发展我国的科学事业默默地奉献.

\section{3 高产著者的年龄分布}

以上从总体的角度分析了第一著者各年龄群体 的论文产出状况. 这里, 我们再从高产著者的角度来 考察学术骨干或学术带头人的年龄结构. 我们分别 统计了 1995 1999 年科学家作为第一著者发表的论 文数量和作为全部著者(既包括第一著者论文, 也包 括合著者论文)发表论文的数量, 并对发表论文最多 的前 100 名著者以他们在 1999 年时所处的年龄段进 行了统计, 得到图 4 和 5 .

首先, 将图 1 与图 4 相比, 两者呈现的双峰态势 是基本一致的. 所不同的是, 图 1 的双峰可明显区分 为主峰和次峰, 而在图 4 中, 双峰表现为均势并立, 青年学者和老年学者遥相呼应. 两图相比, 说明青年 学者在我国整体的科技论文产出能力中已经确立起

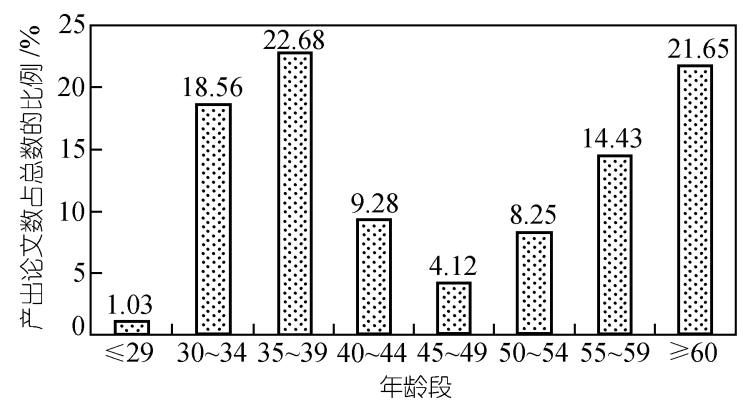

图 4 1994 1999 年发表 CSCD 论文最多的前 100 名著者 的年龄分布(第一著者)

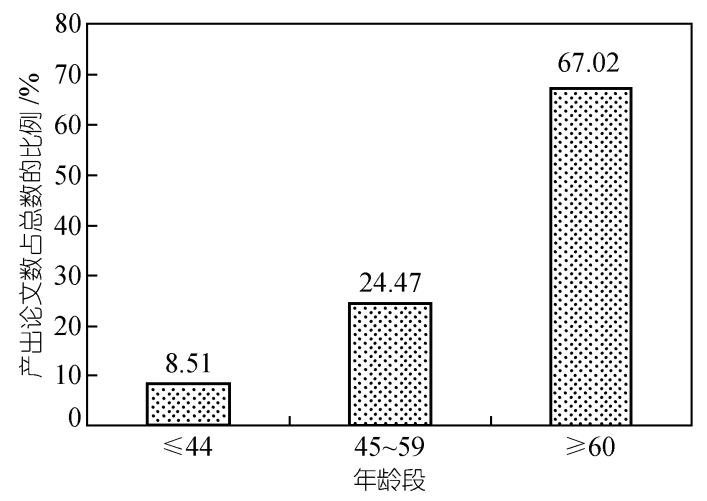

图 $51994 \sim 1999$ 年发表 CSCD 论文最多的前 100 名著者 的年龄分布(全部著者)

了强势地位, 牢固地构筑了属于青年人的论文产出 高峰; 而老年学者虽然在总体论文的年龄分布中处 于衰减群体, 但是在高产著者中, 他们以老一代科学 家坚实的科学素养和丰厚的知识底蕴构筑了中国科 技论文产出力的另一个高峰.

图 5 表明, 在 100 名高产著者(全部著者)中以老 年科学家为主体, 他们绝大多数是博士生导师, 其中 有 26 名院士. 由于图 5 的数据包括了合作论文, 因此, 它的形态可图 4 完全不同: “青年峰”消失, 呈现出论 
文数量随着年龄的增长而增长的情形. 从统计条件 变化而引起的数据和图形变化中, 我们证明了一种 经验性判断: 在科学研究中, 青年科学家往往承担着 具体的科研工作, 包括科学实验和论文撰写等, 因此 在论文署名时多为第一著者. 当我们的统计条件为 第一著者时, 青年科学家便占据了相对的多数, 形成 峰势. 老年科学家往往扮演着指导科研工作的角色, 在论文署名时多为合著者, 因此当我们的统计条件 包括合著论文时, 老科学家的论文产出能力就异峰 突起, 优势明显. Liang 等人 ${ }^{[3]}$ 对我国信息科学领域论 文著者的合作结构所做的分析表明，该领域青年著 者与老年著者的合作频度大于青年著者与青年著者 的合作频度. 这一研究结论与本文的统计现象是完 全吻合的.

从图 1 中我们还观察到, 人才结构曲线的波峰和 波谷形态随着时间的推移而不可逆转地向老年方向 运动. 图 4 和图 5 形态的差异则表明, 人才结构内部 知识的流动方向与人才年龄结构形态变化的运动方 向相反, 老年群体的知识向青年群体流动是人才结 构内部知识流动的主要方向. 20 余年来, 正是新老两 代科学家的合作, 跨越了人才断层的低谷. 到今天, 可 以说已经初步完成了新老交替的历史使命. 然而, 两 图也同时告诉我们一个不争的事实: 高产著者群年 龄老化, 处于高产最佳年龄的中年科学家由于人数 少, 未能形成峰势, 这不能不说是我国科学发展过程 中的一大损失.

\section{4 分析与思考}

\section{1 决定人才结构演变的内因与外力}

人才的成长具有一定规律性, 这是由人才首先 是作为生物人的生理特点所决定的. 如果将图 1 中最 年轻的一端和最年老的一端看作人才结构新陈代谢 的人口和出口, 我们可以观察到一个人才结构演变 的经验性规律：人才结构的形态取决于一定时期内 人口端人才输人量的多少. 当人才输人量明显少于 正常水平, 就会在若干年后的人才结构中逐渐形成 波谷, 严重的话就会造成人才断层. 反之, 人才输人 量明显高于历史平均水平就会逐步形成波峰. 此外, 人才结构的演变过程具有相对稳定、起伏缓慢、不可 逆转的特点. 一旦在人才输人端形成定势, 其形态 (波峰与波谷) 就将长时期保持不变, 直到移至输出端

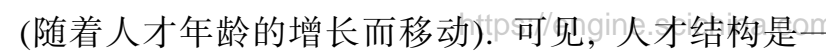

个不断运行和演变的动态机制. 人的生理成长过程 是人才年龄结构演变的内在动力, 而政治、经济、文 化等社会因素是人才结构演变的外部动力. 外部动 力是形成和加速人才结构演变的决定性因素.

在理想条件下, 一个国家科学家队伍的年龄分 布应呈现 “两头小、中间大” 的准正态分布, 且科学 家年龄分布与最佳创造年龄曲线完全重合. 事实上, 由于社会发展诸多因素的影响, 这个曲线会遭受影 响和破坏. 回顾建国 50 余年来我国科学技术发展的 历史, 每一个起落均与科技人才队伍的建设休戚相 关. 新中国建国之初, 一批海外归来的学者成为中国 科学事业的奠基者和第一代创业者. “文革” 前新中 国自己培养的一代知识分子成为改革开放历史时期 内的时代楷模. “文革” 中, 广大热血青年欲学无门, 人才断层的事实就在这样一种无奈中不可逆转地形 成. 改革开放后, 我国奋起直追, 培养了一大批青年 科技人才, 他们继往开来, 正在成为当代中国科学事 业的生力军. 形成 “双峰结构” 的历史背景以及它的 形态缓慢变化, 逐渐从变异结构向理想结构逼近这 样一种现象告诉我们, 社会的人才结构具有脆弱性, 它受制于社会的大环境. 同时, 一个变异的社会人才 结构要想得到缓解, 需要几十年的时间, 要想得到完 全修复则需要几代人的努力.

\section{2 积极的人才政策是我国顺利渡过人才危机的决 定性因素}

大约在 20 多年前, 面对 10 年人才培养的空白, 许多专家看到了人才断层的事实, 并提出了若干年 后可能发生人才危机的预警.

改革开放 20 多年来, 积极的人才政策一直是我 国科技发展政策中的一个重要组成部分．在诸多的 政策措施中最引人注目的就是从中央到地方形成了 面向青年科学家的基金网络. 总理基金(现为国家杰 出青年科学基金)应该说是我国最早设立的面向杰出 青年科学家的专项基金. 此后中央各部委先后设立 了博士后基金、博士点基金、优秀青年教师基金、留 学回国人员基金、百人计划等等, 仅国家自然科学基 金委员会, 除了国家杰出青年科学基金外, 还设有青 年科学基金、优秀中青年人才专项基金、留学人员短 期回国工作讲学专项基金 ${ }^{[4]}$. 各省市也设立了吸引和 培养青年科学家的基金，如上海市青年科技启明星 计划60天津市21 世纪青年科学基金、武汉市青年科 
技晨光计划. 此外, 江苏省、吉林省、重庆市、山西 省、黑龙江省等地都设立了青年科学基金. 基金制度 是我国科技经费分配制度改革的产物. 如此众多的 青年专项基金的涌现烘托出了一个事实：在我国基 金制度形成的过程中，吸引优秀青年人才，为青年人 才成长创造良好的科研环境是我国各级政府长期关 注的一个主题, 并为此作出了持之以恒的不解努力.

根据本项研究的另一组数据统计, 在构筑 “青年 峰” 的这一群体中, 改革开放后培养的博士和硕士在 其中起到了中流砥柱的作用. 在这一年龄段中, 具有 博士和硕士学位的著者的论文占到 $65.44 \%$, 其中有 博士学位的著者的论文占 $44.80 \%$. 另据统计, 30 岁 以下的著者的论文中, 在读和已获得博士、硕士学位 的著者的论文数量占该群体论文总数的 $90 \%$. 这些 数据说明青年人才在改善我国科技人才队伍结构老 化状况的过程中起到了重要作用. 在我们的统计样 本中, 1995 年全部著者的平均年龄是 41.52 岁, 到 1999 年平均年龄降至 38.86 岁. 可见, 一系列积极的 人才政策和教育体制的改革是构筑起本项研究中 “青 年峰” 的主要外部因素. 我国的实践又一次证明社会 政策环境在构建人才队伍结构中的重要作用.

在一定历史时期内，一个国家的科技队伍总有 一个或几个年龄群体处于产出力的旺盛时期, 他们 的文化背景、知识储备、经验积累等因素使他们较之 其他年龄群体有更佳的科研产出力. 随着目前处于 “青年峰” 的高产群体向成熟的中年区域靠近, 他们 将是今后一段时期内我国科学研究能力增量部分的 主要贡献者. 如果能够施之以适宜的政策并配之以 良好的科研条件, 今后的 10 年, 我国的科学技术有 望进人一个快速发展期.

\section{3 对新时期人才流失现象的思考}

如上所述, 10 年 “文革” 造成的人才断层虽然已 经大大地缓解, 但它对我国科学事业发展所产生的 直接和间接的不良影响还将持续相当长的一段时间. 这是历史遗留给人们的惨痛教训. 然而, 我们从图 1 中观察到一个新生的低谷, 应该引起决策者们的关 注和思考.

在新时期中, 人才流动遵循着市场经济的发展 规律. 科技人才的流向受到人才主体价值取向的影 响. 近一个时期以来, 出国热、外企热造成优秀青年 人才的流失是新时期的一种新的社会现象. 30 岁上 下年龄段的著者, 论文产出力出现下滑态势, 已经对
我国科技队伍的年龄曲线形成了新的损坏，这个现 象值得关注. 目前这一新生低谷还尚未形成大的结 构性破坏. 中国要发展, 不能再有大的反复. 管理部 门应该通过统计数据和建立预警系统及时跟踪与预 测人才的变化状况, 做到未雨绸缪, 将问题解决在萌 芽之中, 以防止青年人才流失造成新的人才断层.

\section{4 从全球发展战略出发建立健全一套新的完整的 用人机制和激励机制}

21 世纪是知识经济时代, 综合国力的竞争焦点 越来越突出地表现为人才、智力资源的开发和使用. 谁拥有人才优势, 谁就拥有发展的优势. 有人甚至把 全球人才争夺战喻为一场没有硝烟的战争 ${ }^{[5]}$. 加人 WTO 将使我国处于一个开放的世界经济格局中, 不 可能靠构筑壁垒来遏制科技人才的流失. 因此，科技 队伍的稳定与建设的问题要放在全球的大环境中用 战略的眼光来加以考虑. 按照市场经济规律的要求, 加快建立新的的用人机制和激励机制, 是时代提出 的迫切要求. 在对人才的使用上, 既要遵循人才成长 的一般规律, 又要灵活把握特定时期对人才的特殊 需要, 要根据社会发展的需要和我国的现状来部署 和构筑人才的知识结构、能力结构、专业结构. 随着 老一代科学家的退休, 学术带头人(尖子人才)和具有 战略型科技帅才的选拔应该是各有关部门关注的问 题. 总之, 对于不同的人才群体需要实施不同的政策 与措施, 才能使人才合理使用, 有序流动, 队伍结构 不断优化, 形成一支能够适应 21 世纪全球科技竞争 的人才队伍.

致谢本工作为国家自然科学基金资助项目( 批准号: 79870014).

\section{参考文献}

1 胡小元, 曹秀英, 苏俊彦, 等. 我国优秀科技人才断层问题的调 查. 科技导报, 1993, (1): 30 32

2 赵红州. 科学能力学引论. 北京: 科学出版社, 1984

3 Liang L M, Kretschmer H, Guo Y Z. Age structure of scientific collaboration in Chinese computer science. Scientometrics, 2001, 52(3): 471 486

4 孙枢. 中国国家自然科学基金与科研人才培养. 科学, 1996, 48(6): 4 7

5 刘思峰. 一场没有硝烟的战争——愈演愈烈的全球人才争夺战 与我国的对策. 科学学与科学技术管理, 2000, 21(10): 41 44 